\title{
Properties Prescribing and Control of Direct and Inverse Opal Metamaterials Doped with Spinels, Used for Mems
}

\begin{abstract}
D. IONESCU ${ }^{a, *}$ AND M. KOVACI ${ }^{b}$
a"Gh. Asachi" Technical University of Iasi, Department of Telecommunications, 700506 Iasi, Romania

${ }^{b}$ Politehnica University Timisoara, Department of Communications, 300223 Timisoara, Romania

Properties prescribing and control of the photonic metamaterials operating in microwave fields represents the main task of our study. Magnetic-dielectric composites of opal $\left(\mathrm{SiO}_{2}\right.$ matrices or similar), with insertions of ferrite spinels in the inter-sphere voids $(5 \div 60 \mathrm{~nm}$ inclusions, $3-7$ vol.\%) with insular random/periodic distribution have been considered. Samples have been analyzed by structural simulation, placed inside a rectangular waveguide, mode $T E_{10}$, at frequencies of $20 \div 40 \mathrm{GHz}$, for determining the resonance and antiresonances of the electromagnetic quantities that describe the material behavior. Variations of these quantities have been obtained by changing the structural parameters of the samples and optimal sets of values have been determined for each composite. The domains above the resonant/antiresonant frequency, in which the effective permittivity, respectively permeability are negative have been controlled in order to obtain a wider propagation band. A structure with periodic regular distribution of the inclusions was studied, realized by introducing pre-polarization periodical UV fields $(\lambda=$ $200 \mathrm{~nm}$ ), 2D applied (in cross). Particles molecular coating was applied for maintaining the inclusion positions. The propagation band became tunable by ferrite control and we report a band increasing of about $40 \%$ to $200 \%$, depending on the ions nature in the ferrite and influenced by the structure capacity of interacting with the microwave field.
\end{abstract}

DOI: 10.12693/APhysPolA.135.849

PACS/topics: photonic metamaterials, opal, ferrite inclusions, modulated structure, propagation band control

\section{Introduction}

The 3D photonic metamaterials with artificial opals are used in the bottom up technologies of micromachining for new devices manufacturing, like MEMS applications, optical modulators; optical sensing, miniature antennae, novel waveguides, subwavelength imaging, nanoscale photolithography, photonic circuits, etc. [1-5]. The inverse opal metamaterials are also used for the energy storage and communications, in solar cell technology, for electrochemical energy storage, at supercapacitors, fuel cells, catalysts [1, 4]. Adaptive nanostructured metamaterial structures are difficult to be realized in practice and our study is focused on the design optimization, using and describing of the new phenomena in order to synthesizing the metadevices.

\section{Simulation principles of the adaptive nanomaterial}

The considered structures represent thin films of magnetic-dielectric photonic crystals of direct (a), respectively inverse opal (b), with magnetic inclusions. We have studied: (a) matrices of $\mathrm{SiO}_{2}$ nanospheres (diameter 200-400 nm) with inserted clusters of ferrite spinels (polycrystalline, with spatial dimensions of 5-60 nm and grains of 5$12 \mathrm{~nm}): \mathrm{Mn}_{\mathrm{x}} \mathrm{Co}_{0.6-\mathrm{x}} \mathrm{Zn}_{0.4} \mathrm{Fe}_{2} \mathrm{O}_{4}, \mathrm{Ni}_{\mathrm{x}} \mathrm{Co}_{0.6-\mathrm{x}} \mathrm{Zn}_{0.4} \mathrm{Fe}_{2} \mathrm{O}_{4}$,

*corresponding author; e-mail: danaity@yahoo.com
$\mathrm{La}_{\mathrm{x}} \mathrm{Co}_{0.6-\mathrm{x}} \mathrm{Zn}_{0.4} \mathrm{Fe}_{2} \mathrm{O}_{4}, \quad \mathrm{Nd}_{\mathrm{x}} \mathrm{Co}_{0.6-\mathrm{x}} \mathrm{Zn}_{0.4} \mathrm{Fe}_{2} \mathrm{O}_{4}, \quad(x=$ $0 \ldots 0.5)$ in the inter-sphere voids $(5 \div 60 \mathrm{~nm}$ inclusions, 3-7 vol.\%); (b) matrices of: $\mathrm{SiO}_{2}, \mathrm{TiO}_{2}$ and $\mathrm{CeO}_{2}$, with voids of 100-280 nm (lower at $\mathrm{SiO}_{2}$, higher values for $\mathrm{TiO}_{2}$ ) having spinel inclusion of $\mathrm{Ni}_{0.5} \mathrm{Zn}_{0.5} \mathrm{Fe}_{2} \mathrm{O}_{4}$ or $\mathrm{Co}_{0.5} \mathrm{Zn}_{0.5} \mathrm{Fe}_{2} \mathrm{O}_{4}$, where the giant magnetic antiresonance phenomenon is present $[4,6]$. The lattice constant of opals, $a$, is of thousands of nanometers (e.g.: for $\mathrm{SiO}_{2}$ inverse opal, $a$ is about $2300 \mathrm{~nm}$, with spheres of 400 $600 \mathrm{~nm}$ and void of about $200 \mathrm{~nm}$ ). The ordered structures of opals are illustrated in Fig. 1. The particularities of different methods of introducing inclusions in opal have been considered in our study (impregnation, nanoparticle melts or solutions, etc.), for describing inclusions insular distribution.

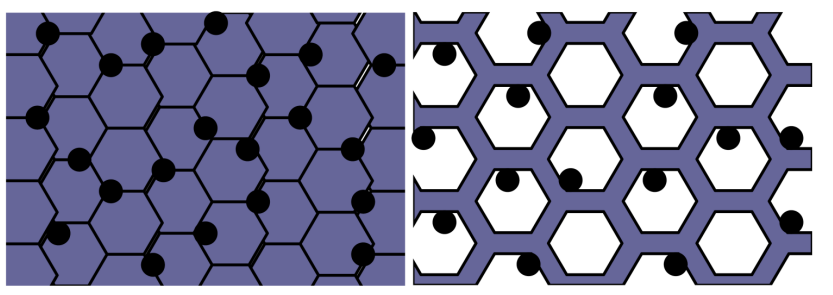

(a)

(b)

Fig. 1. Ordered structure of opals with inclusions: (a) graphical representation of the direct opal structure;

(b) inverse opal.

The ferromagnetic resonance frequency (the maximum of absorption, for the AC field applied at the Larmor frequency) for metamaterial samples can be estimated with the Kittel formula [1, 7]: 
$f_{0[S I]}=\gamma \sqrt{\left(H_{=}+H_{a}\right)\left(H_{=}+H_{a}+M_{S}\right)}$

where $M_{S}[\mathrm{~A} / \mathrm{m}]$ is the saturation magnetization, $H_{a}[\mathrm{~A} / \mathrm{m}]$ is the anisotropy field and $\gamma=2.8 \mathrm{GHz} / \mathrm{kOe}$ is the gyromagnetic ratio. The external bias magnetic field $H_{=}$was taken in range of $0 \div 20 \mathrm{kOe}$.

The ferromagnetic antiresonance occurs when the real part of the magnetic effective permeability for the composite material vanishes: $\mu_{\mathrm{eff}}^{\prime}=0$ and the imaginary part $\mu_{\text {eff }}^{\prime \prime}$ has low a value (an accentuate propagation maximum occurs [4]). The ferromagnetic antiresonance pulsation, $\omega_{F M A R}$ is given by $[4,6]: \omega_{F M A R}=\omega_{M}+\omega_{H \approx}$, where $\omega_{F M A R}=2 \pi f_{F M A R}, \omega_{M}=4 \pi \gamma M_{S}$.

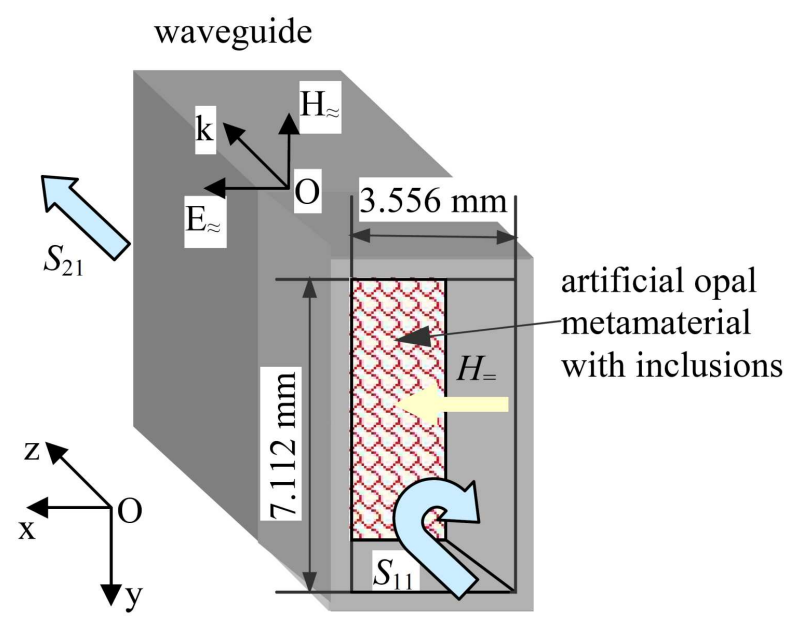

Fig. 2. Simulational set-up: microwave waveguide with the composite material sample, operating in the $T E_{10}$ mode; $H_{=}$is the prepolarizing field of the sample.

The eigenmode solver of the High Frequency Structure Simulator and a proper algorithm proposed by us were used for the resonant/antiresonant frequencies determination. A matrix equation is solved: $\hat{\boldsymbol{S}} \cdot \boldsymbol{x}+k_{0}^{2} \hat{\boldsymbol{T}} \cdot \boldsymbol{x}=\boldsymbol{b}$, where the solver sets the source field vector $\boldsymbol{b}$ to zero. $\hat{\boldsymbol{S}}$ and $\hat{\boldsymbol{T}}$ matrices depend on the geometry and the mesh defined for the finite element method (FEM); $\boldsymbol{x}$ is the electric field vector solution; $k_{0}$ is the free-space wave number corresponding to that $\boldsymbol{x}$ mode and $\boldsymbol{b}$ is the value of the source (field vector) defined for the problem. Equation is solved for different sets of $\left(k_{0}, \boldsymbol{x}\right)$, one for every $\boldsymbol{x}$.

For resonances/antiresonances determination, analysis was performed at $20 \div 40 \mathrm{GHz}$, for metamaterial sample inside a rectangular waveguide [3], propagation mode $T E_{10}$, with a DC pre-polarization field $H_{=}$of $0 \div 20 \mathrm{kOe}$ (Fig. 2). Frequency dependent electromagnetic parameters (electric permittivity $\varepsilon_{\text {eff }}$, magnetic permeability $\mu_{\text {eff }}$, conductivity $\sigma$ ) have been determined. We have focused on the frequency domain where propagation occurs (the refractive index $n>0, k_{2}>0$, while $\varepsilon_{\text {eff }}^{\prime}<0$ and $\left.\mu_{\text {eff }}^{\prime}<0\right)$ and the backward-wave propagation is characteristic for the samples [2,3], domain referred here as propagation band.

\section{Results and discussion}

Frequency dispersion of the effective permeability and permittivity has been represented on parametrical curves and their extreme behavior was observed under the testing field. In Fig. 3 we have given the real part of the permittivity/permeability, represented in the indicated evolution area, for considered material combinations, numbered in Table I. Resonances/ antiresonances can be observed on graphs.

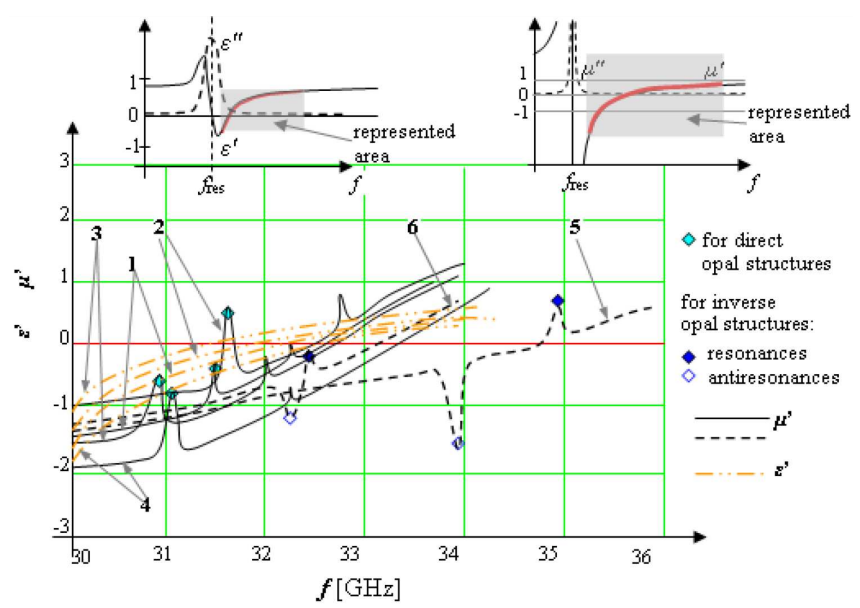

Fig. 3. Frequency dispersion of the effective permeability and permittivity (real parts) for the considered metamaterials with direct/ inverse opals. Resonances/antiresonances can be observed on graphs.

The control parameters have been identified like: inclusions concentration, particle inclusion size, different content of the magnetic ions in the ferrite inclusion, paramagnetic ions in the inclusion ferrite, substrate viscosity, the applied pre-polarizing field, etc.

The resonances analysis indicated us the results given in Table I, obtained from simulation, respectively by theoretical calculation. Phenomenon is used for microwave devices controlled by a magnetic field, working on the basis of resonant or antiresonant phenomena, like attenuators, filters, etc.

We have selected the frequency domains above the antiresonance (to avoid the drop of absorption), starting from its vicinity, in which $\varepsilon_{\text {eff }}^{\prime}$, respectively $\mu_{\text {eff }}^{\prime}$ are negative (Fig. 4). We have studied the possibilities of superimposing these domains, in order to extend the propagation band.

Our studies have demonstrated the widest propagation band for metamaterial structures with an insular distribution of the spinel nanoparticles, periodic arranged in the opal substrate. Pre-polarization periodical UV fields $(\lambda=200 \mathrm{~nm}$ ) have been applied 2D (in cross) for particles leading on their positions corresponding to the field maxima. A molecular coating, magnetic inactive, ensures the particles electrostatic rejection for keeping position. The results obtained with a random versus periodic distribution of the inclusion are presented in Fig. 4. 
TABLE I

Simulation determined values for resonant/antiresonant frequencies for the analyzed metamaterial samples (thin films of opal matrices with ferrite inclusions). Theoretical predicted values are given for comparison

\begin{tabular}{|c|c|c|c|c|c|}
\hline No. & Opal & Ferrite inclusion & $\begin{aligned} f_{\mathrm{FMR}} \text { sim. } & {[\mathrm{GHz}] / } \\
f_{\mathrm{FMR}} \text { th. } & {[\mathrm{GHz}] }\end{aligned}$ & $\begin{array}{c}f_{\text {AFMR sim. }}[\mathrm{GHz}] / \\
f_{\text {AFMR th. }}[\mathrm{GHz}]\end{array}$ & \\
\hline 1 & $\mathrm{SiO}_{2}$ & $\mathrm{Mn}_{x} \mathrm{Co}_{0.6-x} \mathrm{Zn}_{0.4} \mathrm{Fe}_{2} \mathrm{O}_{4}$ & $31.5 / 31.2$ & - & \multirow{4}{*}{$\begin{array}{l}\text { direct opal } \\
\text { structures }\end{array}$} \\
\hline 2 & $\mathrm{SiO}_{2}$ & $\mathrm{Ni}_{x} \mathrm{Co}_{0.6-x} \mathrm{Zn}_{0.4} \mathrm{Fe}_{2} \mathrm{O}_{4}$ & $31.6 / 31.3$ & - & \\
\hline 3 & $\mathrm{SiO}_{2}$ & $\mathrm{La}_{x} \mathrm{Co}_{0.6-x} \mathrm{Zn}_{0.4} \mathrm{Fe}_{2} \mathrm{O}_{4}$ & $30.9 / 30.6$ & - & \\
\hline 4 & $\mathrm{SiO}_{2}$ & $\mathrm{Nd}_{x} \mathrm{Co}_{0.6-x} \mathrm{Zn}_{0.4} \mathrm{Fe}_{2} \mathrm{O}_{4}$ & $31.1 / 30.8$ & - & \\
\hline 5 & $\mathrm{SiO}_{2}$ & $\mathrm{Co}_{0.5} \mathrm{Zn}_{0.5} \mathrm{Fe}_{2} \mathrm{O}_{4}$ & $34.9 / 34.7$ & $33.9 / 33.8$ & \multirow{4}{*}{$\begin{array}{l}\text { inverse opal } \\
\text { structures }\end{array}$} \\
\hline 6 & $\mathrm{SiO}_{2}$ & $\mathrm{Ni}_{0.5} \mathrm{Zn}_{0.5} \mathrm{Fe}_{2} \mathrm{O}_{4}$ & $32.3 / 32.0$ & $31.3 / 31.2$ & \\
\hline 7 & $\mathrm{TiO}_{2}$ & $\mathrm{Co}_{0.5} \mathrm{Zn}_{0.5} \mathrm{Fe}_{2} \mathrm{O}_{4}$ & $23.8 / 23.5$ & $21.4 / 21.1$ & \\
\hline 8 & $\mathrm{CeO}_{2}$ & $\mathrm{Co}_{0.5} \mathrm{Zn}_{0.5} \mathrm{Fe}_{2} \mathrm{O}_{4}$ & $24.9 / 24.7$ & $23.8 / 23.5$ & \\
\hline
\end{tabular}

For this kind of structures, the frequency domain in which the effective electric permittivity is negative could be very wide. The frequency domain in which the effective magnetic permeability is negative being always finite, imposed by the ferrite resonance/antiresonance, the large domain of negative permittivity can ensure a propagation band dependent preponderant on ferrite properties and consequently tunable directly by ferrite control.

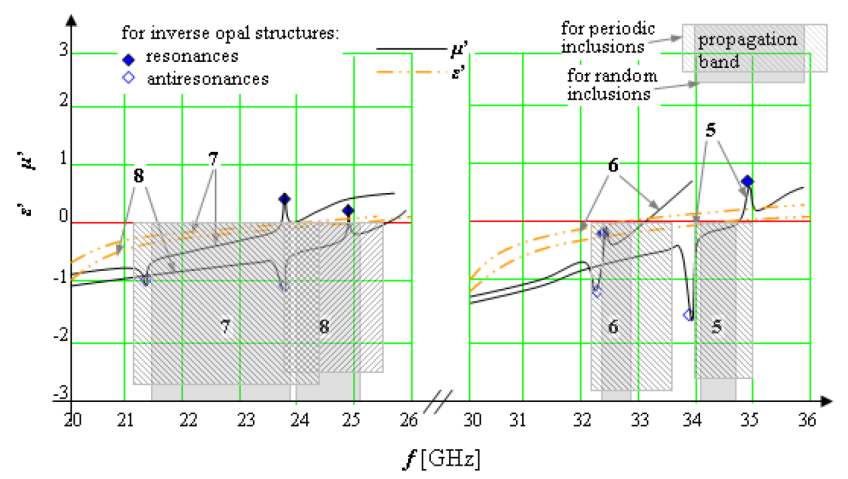

Fig. 4. Illustration of the propagation band at frequencies above the antiresonance for the considered inverse opal metamaterial structures. The materials are denoted with numbers like in Table I.

\section{Conclusion}

The resonant/antiresonant phenomena which represent particular cases of wave propagation through the composed metamaterial samples (opal with ferrite inclusions) have been analyzed by structural simulation. Superimposing of the domains with negative electromagnetic parameters (permeability, permittivity) was enlarged by tuning the control parameters of the structures, internal and external. Optimal results have been obtained for sets of correlated parameters, by an original method developed in our study. E.g. for the $\mathrm{SiO}_{2}$ opal with $\mathrm{Ni}_{\mathrm{x}} \mathrm{Co}_{0.6-\mathrm{x}} \mathrm{Zn}_{0.4} \mathrm{Fe}_{2} \mathrm{O}_{4}$ ferrite inclusions, the recom- mended inclusions concentration is of $4.2 \ldots 4.8 \%$, particle inclusion size (polycrystalline ellipsoidal clusters long axis) of $20 \ldots 30 \mathrm{~nm}$, content of the magnetic ions in the ferrite inclusion $x=0.4$, the applied pre-polarizing field $H_{=}$of $20 \mathrm{kOe}$. Sets of optimal parameter are available for each structure. For the structures with inverse opals, the frequency domains above the antiresonant frequency, starting from its vicinity, in which $\varepsilon_{\text {eff }}$, respectively $\mu_{\text {eff }}$ are negative, superimpose wider when we have an insular periodic distribution of the spinel nanoparticles in the opal substrate. We report an increasing of about $40 \%$ to $200 \%$ for the propagation band, depending on the ions nature in the ferrite and structure capacity of interacting with the microwave field.

\section{References}

[1] Y. Hao, R. Mittra, FDTD Modeling of Metamaterials, Theory and Applications, Artech House, Boston/London 2008.

[2] G.S. Makeeva, O.A. Golovanov, M. Pardavi-Horvath, A.B. Rinkevich, J. Appl. Phys. 113, 173901 (2013).

[3] D. Ionescu, G. Apreotesei, Rom. Rep. Phys. 65, 1328 (2013).

[4] A.B. Rinkevich, D.V. Perov, O.V. Nemytova, Magnetic Antiresonance in Nanocomposite Materials in: Advanced Magnetic and Optical Materials, Eds. A. Tiwari, P.K. Iyer, V. Kumar, H. Swart, John Wiley \& Sons, 2016.

[5] M. Pizzi, V. Konyachkine, V. Lambertini, N. Li Pira, M. Paderi, L. Belforte, M. Pacifico, Informacije MIDEM 36, 177 (2006).

[6] V.V.Ustinov, A.B.Rinkevich, D.V. Perov, M.I. Samoilovich, S.M. Klescheva, J Magn Magn Mater. 324, 78 (2012).

[7] X.C. Tong, Photonic Metamaterials and Metadevices in: Functional Metamaterials and Metadevices, Springer Series in Materials Science, Vol. 262, Cham 2018, p. 71. 